DOI: 10.12957/demetra.2015.14092

\title{
Limiar de reconhecimento do gosto salgado e estimativa de consumo de sódio de transplantados renais
}

\section{Threshold of salty taste recognition and estimate of sodium intake among kidney-transplant recipients}

\author{
Lúcia Chaise Borijes',2 \\ Jéssica Maiara Rosetto ${ }^{1}$ \\ Liandra Martins Garcial \\ 1 Universidade Comunitária da Região de \\ Chapecó, Área de Ciências da Saúde, Curso de \\ Nutrição. Chapecó- SC, Brasil. \\ 2 Universidade Federal de Santa Catarina, \\ Núcleo de Pesquisa de Nutrição em Produção \\ de Refeições, Centro de Ciências da Saúde, \\ Programa de Pós-Graduação em Nutrição. \\ Florianópolis-SC, Brasil.
}

Correspondência / Correspondence Lúcia Chaise Borjes

E-mail: Iborjes@unochapeco.edu.br

\section{Resumo}

Objetivos: O objetivo deste estudo foi avaliar o limiar de reconhecimento do gosto salgado e a estimativa de consumo de sódio dos transplantados renais que frequentam a clínica renal de Chapecó. Métodos: O estudo é de natureza descritiva, com abordagem quantitativa, baseada em estudo de caso. A amostra foi constituída por 52 pacientes, que experimentaram soluções salinas até o reconhecimento da menor concentração de gosto salgado. O limiar foi representado pela média das concentrações em que ocorreram "reconhecimento" e "não reconhecimento". Os demais dados foram coletados dos prontuários. Resultados: A maioria da amostra $(63,5 \%)$ apresentou limiar considerado normal ou abaixo do normal, isto é, menor ou igual a $0,01 \mathrm{~mol} / \mathrm{L}$ $(0,91 \mathrm{~g} / \mathrm{L})$ e o consumo médio de sal foi de $10,7 \mathrm{~g} / \mathrm{dia}$, estimado pelo sódio excretado na urina. Não houve associação significativa entre a classificação dos limiares de reconhecimento do gosto salgado e alguma das variáveis em estudo, quais sejam: idade, sexo, índice de massa corpórea (IMC), pressão arterial, uso de anti-hipertensivos e estimativa do consumo de sal por meio do sódio urinário. No entanto, se avaliada a associação entre a quantidade da menor concentração que reconhecia o gosto salgado com a idade, ela se mostra estatisticamente significativa $\left(\mathrm{r}_{\mathrm{s}}=0,339 ; \mathrm{p}=0,014\right)$. Pacientes com idade mais elevada apresentavam limiares maiores de reconhecimento do gosto salgado. Conclusão: Apesar de serem necessários mais estudos sobre o assunto, observa-se alta estimativa de consumo de sal por meio do sódio urinário, realidade que deve ser tratada com cuidado, sobretudo por pacientes idosos, que apresentam maior limiar de reconhecimento do gosto salgado e podem aumentar 
ainda mais o consumo para tornar o alimento agradável ao seu paladar. Não só os transplantados renais, mas a população brasileira como um todo, demandam ações de saúde pública para reduzir o consumo de sal.

Palavras-chave: Limiar Gustativo. Transplante de Rim. Cloreto de Sódio.

\section{Abstract}

Objectives: The study aimed to evaluate the threshold of salty taste recognition and the estimate of sodium intake among kidney-transplant recipients who attend the renal clinic in Chapecó, Santa Catarina, Brazil. Methods: Study with descriptive nature, quantitative approach, based on a case study. The sample consisted of 52 patients, who tasted saline solutions until recognizing the lowest concentration of salty taste. The threshold was represented by the average value of concentrations where "recognition" and "non-recognition" occurred. Other data were collected from medical records. Results: Most of the sample $(63.5 \%)$ showed a threshold regarded as normal or below normal, i.e. less than or equal to $0.01 \mathrm{~mol} / \mathrm{l}(0.91 \mathrm{~g} / \mathrm{l})$ and the average salt intake was $10.7 \mathrm{~g} /$ day, estimated from urinary sodium excretion. There was no significant association between the classification of salty taste recognition thresholds and some of the variables under study, namely: age, gender, body mass index (BMI), blood pressure, antihypertensive drug use, and estimated salt intake from urinary sodium. However, if evaluating the association between the amount of the lowest concentration recognizing salty taste and age, it shows up as statistically significant $\left(\mathrm{r}_{\mathrm{s}}=0.339\right.$; $\mathrm{p}=0.014)$. Older patients had higher salty taste recognition thresholds. Conclusion: Although there is a need for further research on the subject, a high estimate of salt intake from urinary sodium is observed, and this reality should be carefully addressed, especially by elderly patients, who have a higher salty taste recognition threshold and they may further increase intake in order to make food pleasant to their taste. Not only kidneytransplant recipients, but the Brazilian population as a whole, require public health actions to reduce salt intake.

Key words: Taste Threshold. Kidney Transplantation. Sodium Chloride. 


\section{Introdução}

Um organismo sadio compreende dois rins que atuam na regulação da água, do equilíbrio ácido-básico, dos eletrólitos do organismo e na excreção de produtos do metabolismo através da urina. Além disso, os rins influenciam a pressão sanguínea sistêmica, pela regulação renal. ${ }^{1}$

Na doença renal crônica (DRC), ocorre deterioração progressiva da função dos rins e consequente retenção de substâncias nitrogenadas no sangue. ${ }^{2}$ Quando ocorre falência dos rins, os pacientes são tratados com processos dialíticos enquanto esperam por um transplante renal. ${ }^{3}$ Ocorre que, após o transplante renal, o uso de medicamentos necessários pode causar efeitos colaterais, como a hipertensão. ${ }^{4}$ Não bastasse, a hipertensão arterial sistêmica (HAS) é uma das principais causas da DRC. Nesse contexto, ela pode ser causa e consequência da DRC, sendo que o acontecimento concomitante dessas duas enfermidades é um grande risco para as doenças cardiovasculares. ${ }^{5}$

A propósito, as VI Diretrizes Brasileiras de Hipertensão ressaltam que o consumo abusivo de sal é, claramente, o desencadeante ambiental mais importante de hipertensão arterial. ${ }^{6}$ Uma dieta rica em sódio induz a liberação excessiva de hormônio natriurético, o qual pode aumentar indiretamente a pressão sanguínea. ${ }^{7}$ Por outro lado, o sódio, juntamente com o açúcar e gorduras, é responsável por conferir sabor aos alimentos consumidos diariamente pela população mundial. ${ }^{8}$

A qualidade sensorial é função tanto dos estímulos procedentes dos alimentos como também das condições fisiológicas e sociológicas dos indivíduos que o avaliam, no contexto ambiental em que se localizam esse indivíduo e o próprio produto. ${ }^{9}$

A avaliação da sensibilidade gustativa ao sal é utilizada na área da saúde, bem como em análise sensorial aplicada à Ciência e Tecnologia de Alimentos, para avaliar o paladar e identificar ageusia e hipoageusia de diversas origens, identificando os indivíduos que apresentam risco ou consumo excessivo de sal. ${ }^{10}$ Sendo assim, a concentração de cloreto de sódio necessária para o reconhecimento da salinidade varia em cada indivíduo. Enquanto uma solução é considerada fortemente salgada para um, pode ser insuficiente para o reconhecimento de outro. ${ }^{11}$ Entretanto, de acordo com Guyton \&Hall, ${ }^{12}$ a partir de uma solução de $0,01 \mathrm{~mol} / \mathrm{l}(0,91 \mathrm{~g} /)$, pode-se identificar o gosto de sal. Nilsson ${ }^{13}$ mostrou que a necessidade de soluções mais concentradas que $0,91 \mathrm{~g} / \mathrm{l}$ para identificar o sal é considerada uma alteração na sensibilidade gustativa ao sal.

Os pacientes com DRC ou transplantados renais apresentam alterações no paladar devido às diversas manifestações orais ocorridas pela própria doença ou até mesmo pelo uso de medicamentos. ${ }^{14,15}$ Nesse contexto, o objetivo deste estudo foi avaliar o limiar de reconhecimento do gosto salgado e a estimativa de consumo de sódio dos transplantados renais que frequentam a clínica renal de Chapecó. 


\section{Metodologia}

O estudo é de natureza descritiva, com abordagem quantitativa, baseado em estudo de caso. As pesquisas descritivas têm como objetivo principal a descrição das características de determinado fenômeno e o estudo de caso procura o aprofundamento das questões propostas. ${ }^{16} \mathrm{~A}$ amostra empregada neste experimento foi composta por 52 pacientes transplantados renais, representando 29,88\% do universo de 174 transplantados renais que frequentavam a Clínica Renal em Chapecó em janeiro de 2014. A coleta de dados ocorreu nesse mesmo mês. Foram excluídos os indivíduos com alguma doença aguda, como febre, gripe, dor de garganta, entre outras, durante o período da coleta de dados, além dos menores de 18 anos e que não aceitaram participar do estudo. Num primeiro momento, era perguntado o nome do indivíduo; posteriormente, este era encaminhado individualmente para uma sala onde foi feito o teste de limiar de reconhecimento do gosto salgado.

Para cada participante, foram utilizadas soluções de cloreto de sódio ( $\mathrm{NaCl}$ ) em concentrações crescentes variando entre a menor concentração 0,002 mol/l (0,140 g/l) e a maior concentração de 0,250 mol/l (14,610 g/l). Foram aplicadas quatro gotas da solução-teste, por intermédio de contagotas na ponta da língua do indivíduo, sendo que, após dez segundos sem respirar nem fechar a boca, seguindo a ordem de concentração de $\mathrm{NaCl}$, o indivíduo deveria indicar quando algum estímulo era reconhecido. Neste caso, era repetida a mesma solução. Esta apresentação continuou até que o reconhecimento do gosto salgado ocorresse em duas apresentações sucessivas dentro da mesma série. ${ }^{17}$

O limiar de cada indivíduo é representado pela média das concentrações em que ocorreram "reconhecimento" e "não reconhecimento". ${ }^{18}$ Considerou-se limiar de reconhecimento normal a solução de $0,01 \mathrm{~mol} / \mathrm{l}(0,91 \mathrm{~g} / \mathrm{l})$; as soluções abaixo deste valor foram consideradas abaixo do normal e as soluções acima foram consideradas limiar de reconhecimento acima do normal. ${ }^{12}$

Entre os testes, a boca foi lavada com água destilada e deionizada. Trinta segundos foi o intervalo entre os testes sucessivos. As soluções de $\mathrm{NaCl}$ foram manufaturadas no Laboratório Dietético da Universidade Comunitária da Região de Chapecó (Unochapecó) e acondicionadas em frascos de vidro fechados, em local seco, sem luminosidade e na temperatura ambiente. ${ }^{17}$ Os contagotas não entraram em contato com a língua, para evitar contaminações.

O estudo teve aprovação do Comitê de Ética em Pesquisa da Unochapecó com parecer $n^{\circ}$ $265 / 2013$.

Os demais dados, como idade, sexo, uso de medicamento hipertensivo, pressão arterial, peso, altura e sódio urinário, foram coletados nos prontuários dos pacientes. O índice de massa corporal (IMC) foi construído considerando o quociente entre o peso em quilogramas e a estatura (em 
metros) elevados ao quadrado. A classificação adotada para o IMC de indivíduos com menos de 60 anos foi a classificação proposta pela Organização Mundial da Saúde, ${ }^{19}$ que estabelece: baixopeso <18,50 IMC $\left(\mathrm{Kg} / \mathrm{m}^{2}\right)$; eutrofria 18.50-24.99 IMC $\left(\mathrm{Kg} / \mathrm{m}^{2}\right)$; sobrepeso $\geq 25.00 \mathrm{IMC}\left(\mathrm{Kg} / \mathrm{m}^{2}\right)$; pré-obeso $\geq 25.00$ - 29.99 IMC $\left(\mathrm{Kg} / \mathrm{m}^{2}\right)$; obeso classe I $\geq 30.00-34.99 \mathrm{IMC}\left(\mathrm{Kg} / \mathrm{m}^{2}\right)$; obeso classe II $\geq 35.00$ - 39.99 IMC $\left(\mathrm{Kg} / \mathrm{m}^{2}\right)$; obeso classe III $\geq 40.00 \mathrm{IMC}\left(\mathrm{Kg} / \mathrm{m}^{2}\right)$.

O estado nutricional dos idosos (acima de 60 anos) foi avaliado, segundo pontos de corte propostos pela Organização Pan-Americana da Saúde ${ }^{20}$ no projeto Saúde, Bem-estar e Envelhecimento (SABE), que pesquisou países da América Latina, incluindo o Brasil: Baixopeso: IMC <23 kg/m²; Peso Adequado: IMC 23- 28 kg/m²; Excesso de peso: IMC > 28-30 kg/m²; e Obesidade: IMC $>30 \mathrm{~kg} / \mathrm{m}^{2}$.

O valor ideal de pressão arterial para pessoas com DRC é: <130 x 80 mmHg. ${ }^{6}$ Portanto, consideraram-se níveis pressóricos elevados os indivíduos que apresentaram pressão arterial sistólica $\geq 130$ e/ou pressão arterial diastólica $\geq 80 \mathrm{mmHg}$. Foi considerado hipertenso o indivíduo que apresentou níveis pressóricos elevados e/ou utilizava medicamento anti-hipertensivo.

As variáveis foram descritas por média e desvio-padrão ou mediana e amplitude interquartílica, dependendo da distribuição dessas. As variáveis categóricas foram descritas por frequências absolutas e relativas.

Para comparar médias entre as classificações dos limiares de reconhecimento do gosto salgado, a Análise de Variância (ANOVA) one-way foi aplicada. Em caso de assimetria, o teste de KruskalWallis foi utilizado. Na comparação de proporções, os testes qui-quadrado de Pearson ou exato de Fisher foram aplicados. Para avaliar a associação entre as variáveis contínuas, os testes da correlação linear de Pearson ou de Spearman foram utilizados. O nível de significância adotado foi de $5 \%(\mathrm{p} \leq 0,05)$ e as análises foram realizadas no programa SPSS versão 21.0.

\section{Resultados e discussão}

Do total de 174 transplantados renais que frequentam a clínica, participaram do estudo 52 $(29,88 \%)$ pacientes transplantados. A caracterização desses pacientes está apresentada na tabela 1. 
Tabela 1. Caracterização da amostra de transplantados renais da Clínica Renal. ChapecóSC, 2014.

\begin{tabular}{lc}
\hline \multicolumn{1}{c}{ Variáveis } & $\mathrm{n}=52$ \\
\hline Idade (anos) - média $\pm \mathrm{DP}$ & $45,4 \pm 13,9$ \\
Sexo - n(\%) & $35(67,3)$ \\
Masculino & $17(32,7)$ \\
Feminino & $71,9 \pm 13,7$ \\
Peso (kg) - média \pm DP & $166,7 \pm 8,24$ \\
Altura (cm) - média \pm DP & $25,8 \pm 3,9$ \\
IMC (kg/m $\left.{ }^{2}\right)$ - média DP & \\
Classificação do IMC* - n(\%) & $3(5,8)$ \\
Baixo-peso & $23(44,2)$ \\
Eutrofia & $20(38,5)$ \\
Sobrepeso & $6(11,5)$ \\
Obesidade & $126,9 \pm 12,0$ \\
Pressão Sistólica (mmHg) - média \pm DP & $81,0 \pm 8,7$ \\
Pressão Diastólica (mmHg) - média \pm DP & $44(84,6)$ \\
Níveis pressóricos elevados** - n(\%) & $38(73,1)$ \\
Uso de anti-hipertensivo - n(\%) & $50(96,2)$ \\
Hipertensão*** - n(\%) & $10,7(7-15,7)$ \\
Estimativa do consumo de sal através do sódio & \\
urinário (g/dia) - mediana (Percentis 25-75) & \\
\hline Cono & \\
\hline
\end{tabular}

* Conforme classificação OMS para indivíduos com menos de 60 anos e conforme classificação OPAS para os com idade igual ou superior a 60 anos; ** Pressão Arterial Sistólica $\geq 130$ e/ou Pressão Arterial Diastólica $\geq 80$; *** Considerado hipertenso se apresenta níveis pressóricos elevados e/ou utiliza medicamento anti-hipertensivo. 
A idade média entre os participantes foi de 45,4 anos, com prevalência do sexo masculino, sendo que a maioria se encontra em estado de sobrepeso ou obesidade, totalizando $50 \%$ da amostra.

O ganho ponderal e o desenvolvimento de obesidade são mais frequentes durante os primeiros seis meses após um transplante renal, sendo este aumento entre 12 a $15 \%$ do peso corporal inicial no primeiro ano. ${ }^{21}$ São vários os fatores causais, incluindo o repentino aumento do bem-estar após o transplante renal bem-sucedido, além da liberdade na escolha dos alimentos com menores restrições, assim como a alta ingestão calórica, o estilo de vida sedentário e, principalmente, o aumento do apetite pelo uso de altas doses de esteroides. Também já foi demonstrado que o tratamento crônico com glicocorticoides leva ao aumento da ingestão de alimentos. ${ }^{22}$

Ainda que $73,1 \%$ dos pacientes façam uso de medicação anti-hipertensiva, quase $85 \%$ dos pacientes apresentaram níveis pressóricos elevados. Cabe ressaltar que o paciente, após receber seu enxerto, para evitar a rejeição, necessita de uso de drogas imunossupressoras. E o uso em longo prazo dessas drogas tem como um dos efeitos adversos a hipertensão, que pode variar dependendo da classe dessa droga ${ }^{23}$ Outra justificativa, neste estudo, pode ser o sódio urinário, que apresentou estimativa média do consumo de sal acima do recomendado com 10,7 g/dia. Esse valor é semelhante ao consumo de sal da população em geral, pois segundo a Pesquisa de Orçamentos Familiares (POF), realizada pelo IBGE durante os anos de 2008 e 2009, cada brasileiro consumia em média 11,38 g/dia de sal, sendo o sal de cozinha a principal fonte de ingestão. A Região Sul ficou em terceiro lugar em relação ao maior consumo de sódio diário no Brasil, com 12,91 g/dia de sal, enquanto a OMS recomenda que o consumo não ultrapasse $5 \mathrm{~g} / \mathrm{dia} .^{24,25}$

Segundo o Ministério da Saúde, ${ }^{8,26}$ de acordo com estimativa indireta, calculada a partir da quantidade de sal por habitante comercializada pelas indústrias brasileiras do setor, o consumo de sódio no Brasil ultrapassa o limite máximo recomendado para sua ingestão. A Organização Mundial da Saúde recomenda a redução da ingestão de sódio para reduzir a pressão arterial e o risco de doença cardiovascular, acidente vascular cerebral e doença cardíaca. ${ }^{27}$

Molina e colaboradores ${ }^{28}$ citam, em seu estudo, que o alto consumo de sódio também pode estar relacionado à maior ingestão de alimentos preparados com temperos prontos, sendo esses bastante acessíveis e práticos, além do maior consumo de alimentos industrializados.

Quando avaliada a relação entre prevalências de hipertensão e o nível de industrialização das populações, o estudo Intersalt ${ }^{29}$ evidenciou a associação do consumo de sódio e hipertensão. Populações ocidentais e com alto consumo de sal aparecem possuindo maiores percentuais de HAS, enquanto as populações rurais ou primitivas que não faziam o uso de sal apresentam menores prevalências ou nenhum caso de HAS. 


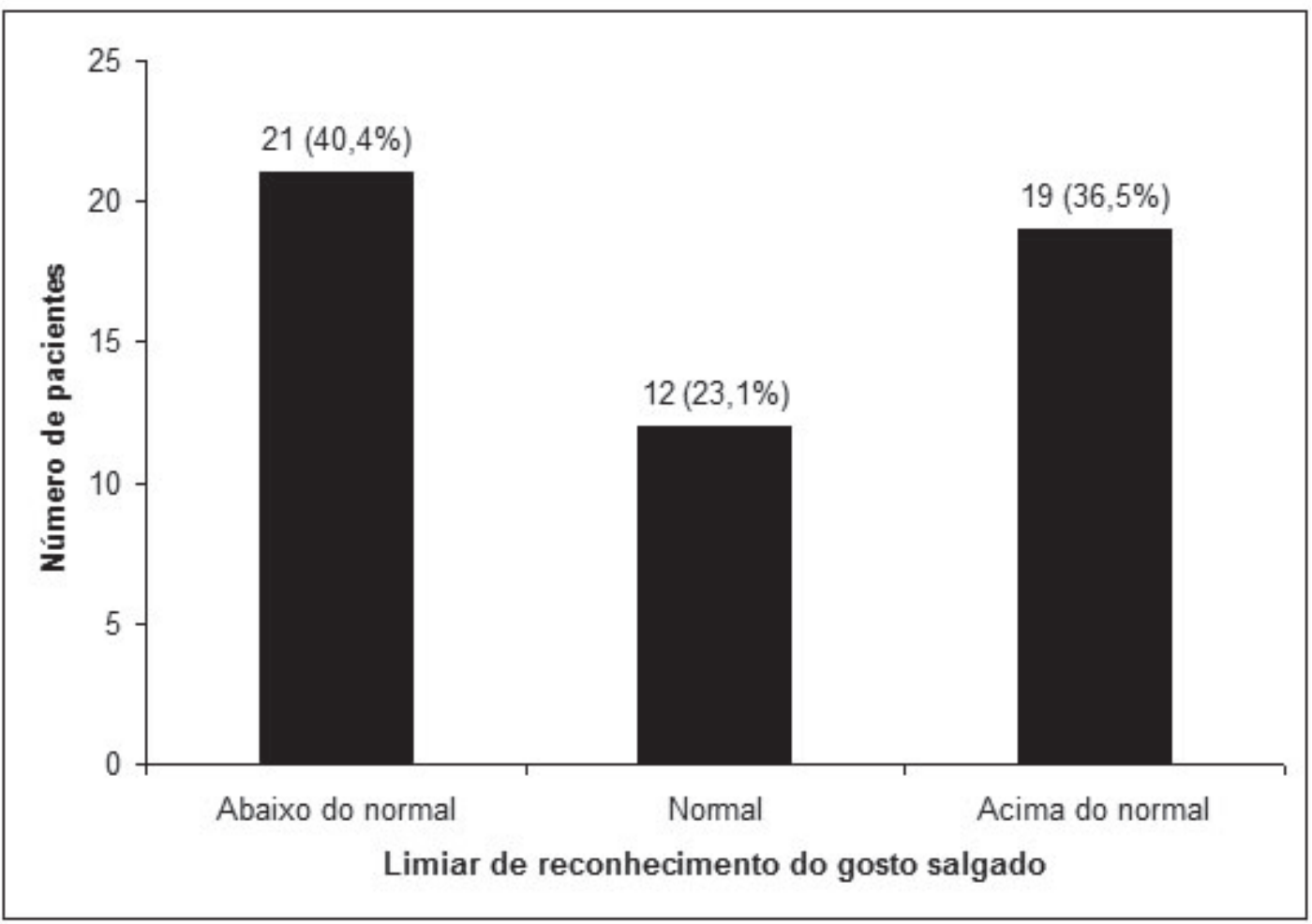

Figura 1. Distribuição dos pacientes quanto ao limiar de reconhecimento do gosto salgado. Chapecó-SC, 2014.

A análise sensorial é a interpretação da análise crítica de determinado alimento utilizando os cinco sentidos; é considerado, portanto, o exame das propriedades sensoriais de um produto. ${ }^{30}$

O limiar de reconhecimento corresponde à concentração de determinado soluto, necessária para promover sua identificação. Tal limiar é geralmente maior que o limiar de detecção. ${ }^{31}$ Enquanto a detecção é a menor concentração em que um estímulo é percebido como sendo diferente da água, o reconhecimento é um conceito estatístico. Por convenção, o valor do limiar refere-se usualmente à concentração em que a mediana dos indivíduos detecta ou $50 \%$ reconhecem. ${ }^{32}$ Com relação a população em estudo, o limiar de reconhecimento do gosto salgado pode ser visto na figura 1 . 
Tabela 2. Associação das variáveis em estudo com o limiar de reconhecimento do gosto salgado. Chapecó-SC, 2014.

\begin{tabular}{|c|c|c|c|c|}
\hline Variáveis & $\begin{array}{l}\text { Limiar abaixo } \\
\text { do normal } \\
\qquad(\mathrm{n}=21)\end{array}$ & $\begin{array}{l}\text { Limiar } \\
\text { Normal } \\
(\mathrm{n}=12)\end{array}$ & $\begin{array}{l}\text { Limiar acima } \\
\text { do normal } \\
\qquad(\mathrm{n}=19)\end{array}$ & $\mathrm{p}$ \\
\hline Idade (anos) - média \pm DP & $40,3 \pm 13,1$ & $45,3 \pm 10,7$ & $50,9 \pm 15,0$ & $0,054 *$ \\
\hline Sexo $-\mathrm{n}(\%)$ & & & & $0,162 * *$ \\
\hline Masculino & $17(81,0)$ & $8(66,7)$ & $10(52,6)$ & \\
\hline Feminino & $4(19,0)$ & $4(33,3)$ & $9(47,5)$ & \\
\hline IMC $\left(\mathrm{kg} / \mathrm{m}^{2}\right)-$ média $\pm \mathrm{DP}$ & $25,7 \pm 3,0$ & $27,0 \pm 4,0$ & $25,1 \pm 4,6$ & $0,427 *$ \\
\hline Classificação do IMC - n(\%) & & & & $0,159 * *$ \\
\hline Baixo peso & $0(0,0)$ & $0(0,0)$ & $3(15,8)$ & \\
\hline Eutrofia & $9(42,9)$ & $5(41,7)$ & $9(47,4)$ & \\
\hline Sobrepeso & $11(52,4)$ & $5(41,7)$ & $4(21,1)$ & \\
\hline Obesidade & $1(4,8)$ & $2(16,7)$ & $3(15,8)$ & \\
\hline $\begin{array}{l}\text { Pressão Sistólica }(\mathrm{mmHg})-\text { média } \\
\pm \text { DP }\end{array}$ & $128,1 \pm 12,5$ & $129,2 \pm 12,4$ & $124,2 \pm 11,2$ & $0,458^{*}$ \\
\hline $\begin{array}{l}\text { Pressão Diastólica }(\mathrm{mmHg})- \\
\text { média } \pm \text { DP }\end{array}$ & $80,0 \pm 8,4$ & $84,2 \pm 9,0$ & $80,0 \pm 8,8$ & $0,353^{*}$ \\
\hline Níveis pressóricos elevados $-\mathrm{n}(\%)$ & $18(85,7)$ & $10(83,3)$ & $16(84,2)$ & $0,982 * *$ \\
\hline Uso de anti-hipertensivo - $\mathrm{n}(\%)$ & $12(57,1)$ & $11(91,7)$ & $15(78,9)$ & $0,076^{* *}$ \\
\hline Hipertensão arterial - n(\%) & $20(95,2)$ & $12(100)$ & $18(94,7)$ & $0,730 * *$ \\
\hline $\begin{array}{l}\text { Estimativa do consumo de sal } \\
\text { através do sódio urinário (g/dia) - } \\
\text { média } \pm \text { DP }\end{array}$ & $11,3(6-17)$ & $11,6(9-15)$ & $9,9(7-12)$ & $0,626^{* * *}$ \\
\hline
\end{tabular}

* Análise de Variância (ANOVA) one-way; ** Teste qui-quadrado de Pearson; *** Teste de Kruskal-Wallis.

Não houve associação significativa entre a classificação dos limiares de reconhecimento do gosto salgado com nenhuma das variáveis em estudo (tabela 2). No entanto, se avaliada a associação da quantidade da menor concentração que reconhecia o gosto salgado com a idade, a associação é estatisticamente significativa $\left(r_{s}=0,339 ; p=0,014\right)$, conforme demonstra a figura 2. Pacientes com idade mais elevada apresentavam limiares maiores de reconhecimento do gosto salgado. 
Ocorre que, acima de 60 anos há uma redução no número de botões gustativos, responsáveis pelo sentido do paladar, o que ocasiona alterações no limiar gustativo, ou seja, é necessária uma maior concentração de doce, salgado, ácido e amargo para que o idoso possa distinguir esse gosto da água e distinguir os sabores. A dificuldade do idoso em detectar o gosto salgado dos alimentos o predispõe a salgar mais a comida., ${ }^{9,3}$

Os sentidos do olfato e paladar, ligados à degustação de preparações, ficam bastante reduzidos no envelhecimento, além de se tornarem menos eficientes, provocando inadequações no processo de ingestão de alimento prejudiciais, como mais sal e açúcares, prejudicando a saúde. ${ }^{34}$

Em estudo realizado na Universidade de Brasília, em 2008, constatou-se que os idosos precisaram de aproximadamente 25 vezes mais sal do que os adultos para detectar a presença desta substância. Os adultos detectaram o gosto salgada a partir de soluções com 0,010\% de sal ( $\mathrm{NaCl}$ ), mas os idosos só detectaram o gosto salgado a partir das soluções com $0,266 \%$ de sal $(\mathrm{NaCl}) .{ }^{35}$

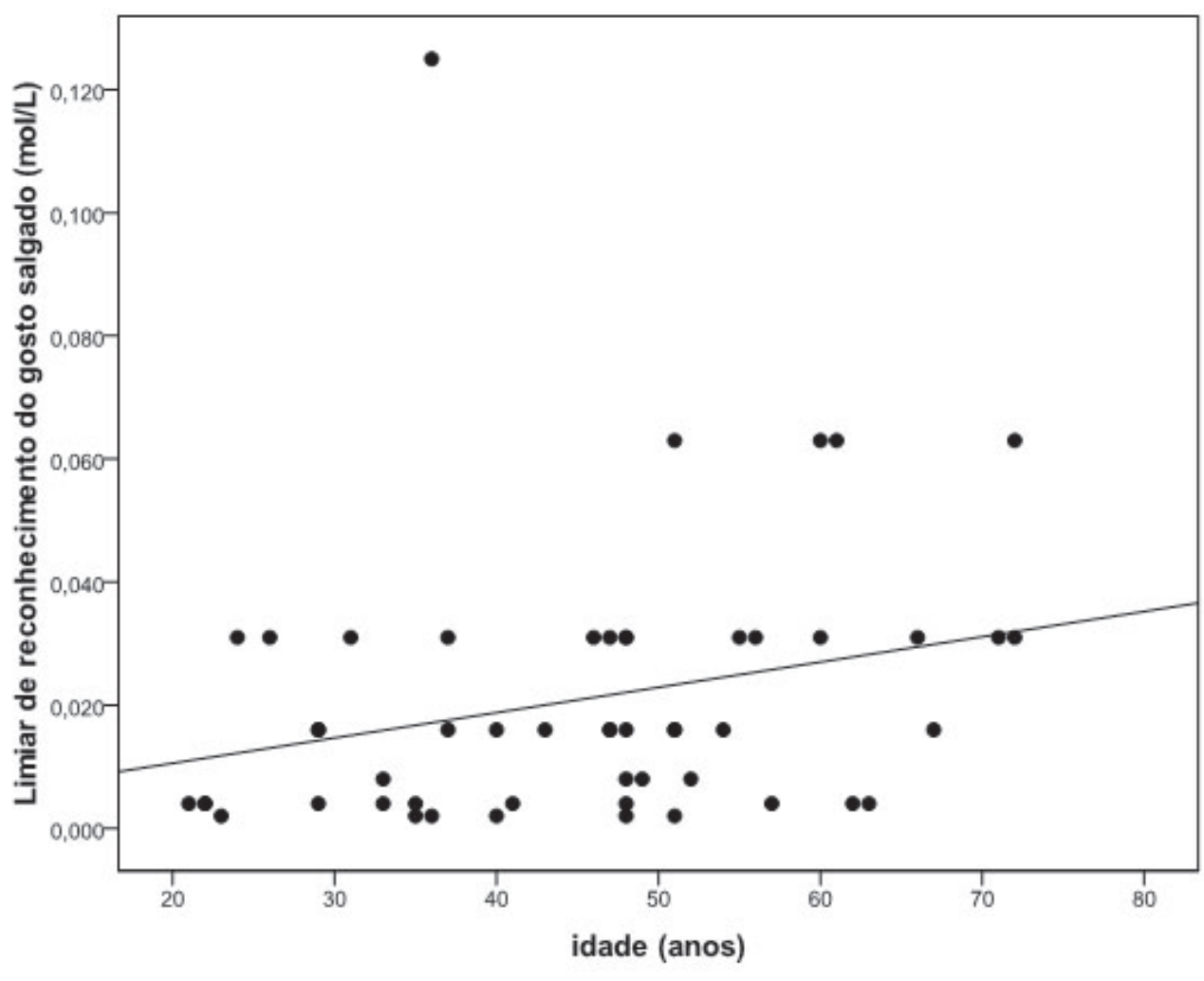

Figura 2. Associação entre a idade e o limiar de reconhecimento do gosto salgado. ChapecóSC, 2014. 
Apesar de não significativo $(p=0,135)$, pacientes em uso de medicação anti-hipertensiva apresentaram maior estimativa do consumo de sal em gramas por dia através do sódio urinário do que os que não utilizavam medicação [10,9 (8-16) g/dia vs 8,4 (4-14) g/dia, respectivamente].

Nem todos os indivíduos apresentam sensibilidade ao sal e, portanto, nem todos desenvolvem hipertensão quando expostos a elevado consumo de sal. Por motivos não esclarecidos, tem sido sugerida a incapacidade renal de excreção da sobrecarga salina com consequente aumento da volemia e/ou ativação simpática, com consequente vasoconstrição. ${ }^{36}$

Perin e colaboradores ${ }^{37}$ mostraram que o consumo médio de sal de uma população hipertensa

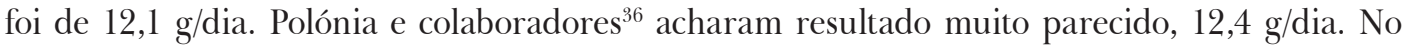
presente estudo, a estimativa do consumo de sal pelo sódio urinário foi de 10,7 g/dia, para hipertensos e normotensos.

Quando a ingestão de sódio excede as necessidades do organismo, existem mecanismos que excretam o excedente para manutenção da normalidade. No entanto, há um limite até o qual se pode eliminar sódio, e uma ingestão superior provoca aumento no volume de sódio e consequente retenção de água, aumento da volemia e da pressão arterial. ${ }^{38}$

Estudos demonstram a existência de relação direta entre excreção urinária de sódio e a pressão arterial média. Elevações da pressão arterial levam ao aumento imediato da diurese e da perda renal de sódio, fenômeno conhecido como natriurese pressórica. ${ }^{39-41}$ No entanto, em relação aos níveis pressóricos estarem acima ou abaixo do considerado normal, a estimativa do consumo de sal foi semelhante entre os grupos [10,8 (7-15) g/dia vs 10,3 (6-12) g/dia, respectivamente; $\mathrm{p}=0,645]$. Essa ocorrência pode ser explicada pelo fato de alguns indivíduos excretarem maiores quantidades de sódio sem um aumento na pressão arterial, enquanto outros não. ${ }^{42}$

A resposta da pressão arterial em relação ao consumo de sódio acontece de maneira diferente para cada indivíduo, existindo os que manifestam tendências maiores a oscilações, tanto na restrição, quanto na adição de sal à dieta, fator conhecido como sensibilidade ao sal. ${ }^{43}$

Por fim, o uso de anti-hipertensivo não se relacionou significativamente com os níveis pressóricos estarem acima ou abaixo do considerado normal (72,7\% vs $75 \%$; $=1,000)$. Novamente fazendo referência ao estudo de Perin e colaboradores ${ }^{37}$, podemos afirmar que mesmo em pacientes com o uso de cerca de três classes de medicamentos anti-hipertensivos, os níveis de pressão arterial observados indicam controle insatisfatório da pressão arterial. 


\section{Conclusões}

Não houve associação significativa dos limiares de reconhecimento do gosto salgado com nenhuma das variáveis em estudo, quais sejam: idade, sexo, IMC, pressão arterial, uso de anti-hipertensivos e estimativa do consumo de sal através do sódio urinário. A maioria $(63,5 \%)$ apresentou limiar considerado normal ou abaixo do normal, isto é, menor ou igual a $0,01 \mathrm{~mol} / \mathrm{L}$ $(0,91 \mathrm{~g} / \mathrm{L})$.

O uso de medicamentos anti-hipertensivos não se relacionou com os níveis pressóricos estarem acima ou abaixo do recomendado. Ainda que $73 \%$ da amostra fizessem uso destes medicamentos, $85 \%$ apresentaram pressão arterial elevada.

A média de consumo de sal, estimada pelo sódio excretado na urina, foi 10,7 g/dia. Por estudos anteriores, pode-se notar relação entre sua ingestão e HAS. Mesmo que não significativo, a maior estimativa de consumo de sal foi em pacientes hipertensos sob medicação. Apenas apresentou significância estatística a menor concentração de reconhecimento do gosto salgado com a idade. Os idosos são os maiores prejudicados, necessitando de maiores quantidades para reconhecer o sabor.

Apesar de serem necessários mais estudos sobre o assunto, salienta-se o consumo excessivo de sal, realidade que precisa ser tratada com cuidado. Não só os transplantados renais, mas a população brasileira, necessitam de ações de saúde pública com o objetivo de reduzir o consumo de sal.

\section{Referências}

1. Aumüller G, Aust G, Doll A, Engele J, Kirsch J, Mense S, et al. Anatomia. Rio de Janeiro: Guanabara Koogan; 2009.

2. Teixeira Neto F. Nutrição clínica. Rio de Janeiro: Guanabara Koogan; 2003.

3. Silverman S, Eversole IR, Truelove EL. Fundamentos de medicina oral. Rio de Janeiro: Guanabara Koogan; 2004.

4. Brasil. Ministério da Saúde. Dicas de saúde: dieta transplantado renal [Internet]. Biblioteca Virtual em Saúde do Ministério da Saúde. 2008. Acesso em: 18 out. 2014. Disponível em: http://bvsms.saude. gov.br/bvs/dicas/143dieta_transplante_rim.html

5. Bortolotto LA. Hipertensão arterial e insuficiência renal crônica. Rev. Bras. Hipertens. 2008; 15(3):152-155.

6. Sociedade Brasileira de Cardiologia; Sociedade Brasileira de Hipertensão; Sociedade Brasileira de Nefrologia. VI Diretrizes Brasileiras de Hipertensão. Arq. Bras. Cardiol. 2010; 95(1):1-51.

7. Brêtas ACP, Gamba MA. Enfermagem e saúde do adulto. São Paulo: Manole; 2006.

8. Brasil. Ministério da Saúde. Guia alimentar para a população brasileira: promovendo a alimentação saudável. Brasília: CGPAN; 2005. 
9. Monteiro MAM. Percepção sensorial dos alimentos em idosos. Espaç. Saúde 2009; 10(2):34-42.

10. Piovesana PM, Gallani MCBJ, Sampaio KL. Revisão: metodologias para análise da sensibilidade gustativa ao sal. Braz. J. Food Technol. 2012; 15(3):182-190.

11. Hayes JE, Sullivan BS, Duffy VB. Explaining variability in sodium intake through oral sensory phenotype, salt sensation and liking. Physiol. Behav. 2010; 100(4):369-380.

12. Guyton AC, Hall JE. Tratado de fisiologia médica. 12. ed. Rio de Janeiro: Elsevier; 2011.

13. Nilsson B. Taste of acuity of human palate. Studies with taste solutions on subjects in different age groups. Acta Odontol. Scand. 1979; 37(4):235-52.

14. Reeves J. oral health problems in the renal patient. Dental Nursing 2008; 4:618-21.

15. Proctor R, Kumar N, Stein A, Moles D, Porter S. Oral and dental aspects of chronic renal failure. J. Dent. Res. 2005; 84:199-208.

16. Gil AC. Como elaborar projetos de pesquisa. São Paulo: Atlas; 2003.

17. Antonello VS, Antonello ICF, Santos CAL. Sensibilidade gustativa ao sal, natriúria e pressão arterial em indivíduos normotensos. Rev. Assoc. Med. Bras. 2007; 53(2):142-146.

18. Associação Brasileira de Normas Técnicas. NBR 13172: teste de sensibilidade em análise sensorial. Rio de Janeiro: ABNT; 1994.

19. World Health Organization. Diet, nutrition and the prevention of chronic diseases. Report of a Joint WHO/FAO Expert Consultation. Geneva: WHO; 2003. WHO Technical Report Series No. 916.

20. Wold Health Organization. Encuesta multicentrica: salud, bien estar y envejecimiento (SABE) en América Latina y el Caribe. In: Anales da $36^{a}$ Reunión del Comité Asesor de Investigaciones en salud. Washington, D. C.: Wold Health Organization; mayo 2001.

21. Lienert RSC, Figueiredo CEP, Figueiredo AEPL. Evolução do peso durante o primeiro ano de transplante renal e a ocorrência de Diabetes Mellitus após 5 anos de seguimento. Rev Ciência \& Saúde 2014; 7(3):148-154.

22. Sharif A. Metabolic syndrome and solid-organ transplantation. Am. J. Transplant 2010; 10(1):12-17.

23. Boots JM, Christiaans MH, van Hooff JP. Effect of immunosuppressive agents on long-term survival of renal transplant recipients: focus on the cardiovascular risk. Drugs 2004; 64:2047-73.

24. Associação Brasileira das Indústrias da Alimentação. Cenário do consumo de sódio no Brasil: um estudo realizado com base em dados do IBGE. São Paulo: ABIA; 2013.

25. Sarno F, Claro RM, Levy RB, Bandoni DH, Monteiro CA. Estimativa de consumo de sódio pela população brasileira, 2008-2009. Rev. Saúde Pública 2013; 47(3):571-8.

26. Brasil. Ministério da Saúde. Hipertensão arterial sistêmica para o Sistema Único de Saúde. Brasília: Ministério da Saúde; 2006.

27. World Health Organization. The world health report 2002: reducing risks, promoting healthy life. Geneva: WHO; 2002.

28. Molina MCB, Cunha RS, Herkenhoff LF, Mill JG. Hipertensão arterial e consumo de sal em população urbana. Rev. Saúde Púb. 2003; 37(6):743-50. 
29. Intersalt Cooperative Research Group. Intersalt: an international study of electrolyte excretion and blood pressure. Results for 24 hour urinary sodium and potassium excretion. BMJ 1988; 297(6644):319-328.

30. Cecchi HM. Fundamentos teóricos e práticos em análise de alimentos. 2. ed. Campinas: Editora UNICAMP; 2003.

31. Meilgaard M, Civille GV, Carr BT. Sensory evaluation techniques. 3. ed. Boca Raton: CRC; 1999.

32. Bartoshuk L. The psychophysics of taste. AJCN 1978; 31(6):1068-77.

33. Lima JP. A influência das alterações sensoriais na qualidade de vida do idoso. Rev. Científica Eletrônica de Psicologia 2007; 5(4):01-8.

34. Ortiz NCL. El gusto por el sabor salado. Persp. Nutr. Hum. 2014; 16(1):99-109.

35. Correia JHC. Redução do paladar em idosos para o gosto doce e salgado. [Monografia]. Brasília: Universidade de Brasília; 2008.

36. Polónia J, Maldonado J, Ramos R, Bertoquini S, Duro M, Almeida C, et al. Determinação do consumo de sal numa amostra da população portuguesa adulta pela excreção urinária de sódio: sua relação com rigidez arterial. Rev. Port. Cardiol. 2006; 25(9):801-17.

37. Perin MS, Cornélio ME, Rodrigues RCM, Gallani MCBJ. Caracterização do consumo de sal entre hipertensos segundo fatores sociodemográficos e clínicos. Rev. Latino-Am Enfermagem 2013; 21(5):1-9.

38. Viegas C. Sal e doença cardiovascular. Rev. Fact Risco 2008; (10):12-8.

39. Oparil S. Low sodium intake: cardiovascular health benefit or risk? N. Engl. J. Med. 2014; 371(7):677-9.

40. Gonçalves ARR, Zatz R, Heimann JC. O papel do rim no controle da pressão arterial. Hipertensão $2000 ; 3(1): 6-14$.

41. Heimann JC, Vidonho Junior AF, Ruivo GF. Mecanismos hipertensores em indivíduos portardores de nefropatia crônica. Rev. Bras. Hipertens. 2002; 9(2):135-40.

42. Cowley AW. Genetic and nongenetic determinants of salt sensibility and blood pressure. Am. J. Clin. Nutr. 1997; 6(Suppl):587-93.

43. Graudal N, Jürgens G, Baslund B, Alderman MH. Compared with usual sodium intake, low- and excessive-sodium diets are associated with increased mortality: a meta-analysis. Am. J. Hypertens. 2014; 27(9):1129-37.

Recebido: 15/12/2014

Revisado: 07/3/2015

Aprovado: 25/3/2015 